\title{
Irisin relaxes rat thoracic aorta through inhibiting signaling pathways implicating protein kinase $\mathrm{C}$
}

\author{
Sadettin DEMIREL*(D), Serdar ŞAHİNTÜRK (D), Naciye IŞBIIL (D), Fadıl ÖZYENER (D) \\ Department of Physiology, Faculty of Medicine, Bursa Uludağ University, Bursa, Turkey
}

Received: $10.05 .2021 \quad \bullet \quad$ Accepted/Published Online: 04.11.2021 • Final Version: 14.04 .2022

Background/aim: Irisin, a newly identified exercise-derived myokine, has been found involved in a peripheral vasodilator effect. However, little is known regarding the potential vascular activity of irisin, and the mechanisms underlying its effects on vascular smooth muscle have not been fully elucidated. This study was aimed to investigate the effects of irisin on vascular smooth muscle contractility in rat thoracic aorta, and the hypothesis that protein kinase $\mathrm{C}(\mathrm{PKC})$ may have a role in these effects.

Materials and methods: Isometric contraction-relaxation responses of thoracic aorta rings were measured with an isolated organ bath model. The steady contraction was induced with $10 \mu \mathrm{M}$ phenylephrine (PHE), and then the concentration-dependent responses of irisin (0.001-1 $\mu \mathrm{M}$ ) were examined. The time-matched vehicle control (double distilled water) group was also formed. To evaluate the role of PKC, endothelium-intact thoracic aorta rings were incubated with $150 \mathrm{nM}$ bisindolylmaleimide I (BIM I) for 20 min before the addition of $10 \mu \mathrm{M}$ PHE and irisin. Also, a vehicle control group was formed for dimethyl sulfoxide (DMSO).

Results: Irisin exerted the vasorelaxant effects at concentrations of $0.01,0.1$, and $1 \mu \mathrm{M}$ compared to the control group ( $\mathrm{p}<0.001$ ). Besides, PKC inhibitor BIM I incubation significantly inhibited the relaxation responses induced by varying concentrations of irisin ( $\mathrm{p}: 0.000$ for 0.01 $\mu \mathrm{M}$; p: 0.000 for $0.1 \mu \mathrm{M}$; p: 0.000 for $1 \mu \mathrm{M}$ ). However, DMSO, a solvent of BIM I, did not modulate the relaxant effects of irisin (p > 0.05).

Conclusion: In conclusion, physiological findings were obtained regarding the functional relaxing effects of irisin in rat thoracic aorta. The findings demonstrated that irisin induces relaxation responses in endothelium-intact thoracic aorta rings in a concentration-dependent manner. Furthermore, this study is the first to report that irisin-induced relaxation responses are regulated probably via activating signaling pathways implicating PKC.

Key words: Irisin, rat thoracic aorta, vasodilation, protein kinase $\mathrm{C}$

\section{Introduction}

Irisin, a newly identified hormone-containing 112 amino acids, which is secreted mainly by skeletal muscle cells during exercise, is a cleavage product of fibronectin type III domain-containing protein 5 (FNDC5) [1]. Irisin increases mitochondrial biogenesis and energy expenditure by activating the conversion of white adipocytes to brown adipocytes $[1,2]$. Circulating levels of irisin are associated with obesity, type II diabetes mellitus, insulin resistance, hypertension, and atherosclerosis. Recently, one of the agents predicted to be used in the prevention, follow-up, and treatment of these metabolic diseases and/or pathologies is the irisin molecule [3-5].

Vasoreactivity is of fundamental importance in various cardiovascular diseases such as hypertension, headache, and stroke [6]. Although some studies have demonstrated the peripheral vasodilator effect of irisin, little is known about the potential vascular activity of this molecule,

* Correspondence: sdemirel@uludag.edu.tr and the mechanisms underlying its effects on vascular smooth muscle contraction-relaxation responses have not been fully elucidated yet. Besides, studies with this newly discovered adipomyokine have had several conflicting results. Therefore, more studies are needed to demonstrate the functional effects of irisin on vascular tissue and the mechanisms involved in these effects.

In the present study, it was aimed to investigate the effects of irisin on vascular smooth muscle contractility on rat thoracic aorta, a conducting artery, and the possible roles of protein kinase $\mathrm{C}$ (PKC) and dimethyl sulfoxide (DMSO), a solvent of PKC inhibitor bisindolylmaleimide I (BIM I).

\section{Materials and methods}

\subsection{Ethics}

This study was approved by the decision of Bursa Uludağ University Animal Experiments Local Ethics Committee dated 03/04/2019 and numbered 2019-04/10. 


\subsection{Test animals}

In this study, male Wistar Albino rats (10-12 weeks of age), obtained from Bursa Uludağ University Experimental Animals Breeding Application and Research Center, were used. All experiments were performed according to the National Institutes of Health Guidelines on the Use of Laboratory Animals.

\subsection{Chemicals and reagents used in experiments}

Irisin, BIM I, phenylephrine hydrochloride (PHE), acetylcholine chloride (ACh), DMSO, and chemicals required to prepare Krebs solution used in the experiments were purchased from Sigma Aldrich (St Louis, MO, USA). Irisin, $\mathrm{PHE}$, and ACh were dissolved in distilled water; BIM I was dissolved in DMSO.

\subsection{Isolated organ bath experiments}

\subsubsection{Preparation of aortic rings and mounting in organ} baths

The animals used in the experiments were decapitated without anesthesia, taking care that they were not exposed to any stress factors. The thoracic cavity of the animals was opened rapidly and then the thoracic aorta was gently removed and placed in a Petri dish containing $0-4{ }^{\circ} \mathrm{C}$ Krebs solution. The Krebs solution contained: $\mathrm{NaCl} 119.0 \mathrm{mM}$, $\mathrm{KCl} 4.7 \mathrm{mM}, \mathrm{MgSO}_{4} 1.5 \mathrm{mM}, \mathrm{KH}_{2} \mathrm{PO}_{4} 1.2 \mathrm{mM}, \mathrm{CaCl}_{2}$ $2.5 \mathrm{mM}, \mathrm{NaHCO}_{3} 25.0 \mathrm{mM}$, and glucose $11.0 \mathrm{mM}$. The isolated vessels were cleared of adhering fat and connective tissue and then divided into $2-3 \mathrm{~mm}$ rings. Thoracic aorta rings were connected to the isometric force transducer (SS12LA force transducer, BIOPAC Systems, Inc. Aero Camino, USA) of the four-chamber organ bath (Commat Ltd., Ankara, Turkey) through steel wires as rapidly as possible to measure the isometric contractile responses. The temperature of the organ bath was kept constant at $37{ }^{\circ} \mathrm{C}\left( \pm 0.5{ }^{\circ} \mathrm{C}\right)$ through a thermocirculator containing distilled water. The organ bath chambers were oxygenated with $95 \% \mathrm{O}_{2}-5 \% \mathrm{CO}_{2}$ mixed gas during the experiment to maintain a $\mathrm{pH}$ of 7.4. Briefly, the isolated organ bath model was used as previously described [7].

\subsubsection{Vessel viability testing}

Before each experiment, aortic rings were loaded with 2 $\mathrm{g}$ of resting tension and then stabilized for $60 \mathrm{~min}$. The Krebs solution, containing the tissues, was refreshed every 15 min during the equilibration period. After this period, vessel viability and endothelial integrity were estimated by its stable and reproducible response to the addition of $10^{-5} \mathrm{M}$ PHE and $10^{-5} \mathrm{M}$ ACh. Those with $>60 \%$ relaxation against ACh among the vascular segments were accepted as endothelium intact. Then, active, inhibitor, and vehicle substance applications were precisely performed into Krebs solution using an adjustable automatic pipette.

\subsubsection{Preliminary experiments}

First, in preliminary experiments, PHE was added to isolated organ bath chambers in cumulative concentrations (0.0001-100 $\mu \mathrm{M})$ after the equilibration period to determine the submaximal contraction dose. In preliminary experiments, it was also investigated that whether irisin, BIM I, and DMSO at the concentrations used in the study have significant effects on basal tone on rat thoracic aorta rings.

2.4.4. Measurements of vascular reactivity in isolated thoracic aortas

Cumulative concentrations (0.001-1 $\mu \mathrm{M})$ of irisin were used in all experimental groups. $10^{-5} \mathrm{M}$ PHE was administrated to induce a steady contraction in the aortic rings, and then the concentration-dependent responses of irisin were examined to determine the vasodilative effect of irisin on the endothelium-intact thoracic aorta (control group). The time-matched vehicle control (double distilled water) group was also formed.

To evaluate the role of PKC, endothelium-intact thoracic aorta rings were incubated with $150 \mathrm{nM}$ BIM I (PKC inhibitor) for $20 \mathrm{~min}$ before the addition of $10 \mu \mathrm{M}$ $\mathrm{PHE}$ and irisin.

Following the incubation of inhibitor used for mechanism research, the responses developed depending on cumulative concentrations of irisin were compared with the control group, and then the differences between the relaxation amplitudes were calculated. Also, a "vehicle" control group was formed for DMSO, which was used as a solvent.

Eight aortic preparations were used for each of the experimental groups formed according to the active, inhibitor, or vehicle agents used in the study.

Contraction-relaxation responses were measured isometrically by force transducer on the MP36 recording system (BIOPAC Systems, Inc. Aero Camino, USA). The records obtained were evaluated in a computer environment with Biopac Student Lab 3.7.7 program (BIOPAC Systems, Inc. Aero Camino, USA).

\subsection{Statistical analysis}

The size of the difference between the groups was calculated by using Cohen's $d$ statistics for the comparison of the independent means and the effect size was determined as 0.30 . While the power of the study was 0.80 and the alpha value was 0.05 , the minimum sample size required was estimated at $n=5$ per group. Power analysis was done under GPower 3.1. SPSS v.23.0 program (SPSS Inc., Chicago, IL, USA) was used for statistical analysis. Test of normality was determined by Shapiro Wilk test. The data were expressed as 'Mean \pm SD (Standard Deviation)' for parametric data. The statistical significance between the two independent groups was analyzed using the independent sample t-test. One-way ANOVA was used for multiple comparisons. Dunnett's T3 post hoc test was used to determine the differences between groups. For groups without the assumption of normality, Mann-Whitney U 
and Kruskal-Wallis with Dunn's post-hoc test were used. $P$ values of $<0.05$ were considered statistically significant.

\section{Results}

In preliminary experiments, the submaximal contraction response was taken against $10 \mu \mathrm{M}$ PHE. In all subsequent experiments, precontractions were induced with the addition of $10 \mu \mathrm{M}$ PHE. Stable and reproducible contraction responses occurred in rat thoracic aorta rings with the submaximal concentration of PHE. In preliminary experiments, it was also determined that irisin, BIM I, and DMSO at the concentrations used in the study had no significant effects on basal tone in rat thoracic aorta rings.

\subsection{Irisin induced vasodilation in rat thoracic aortas}

Cumulatively applied irisin induced concentrationdependent relaxation responses on endothelium-intact rat thoracic aorta rings precontracted with PHE. The relaxing effect of irisin was found to be significantly higher at concentrations of $0.01,0.1$, and $1 \mu \mathrm{M}$ compared to the control group (double distilled water-treated rat thoracic aorta rings) (p: 0.589 for $0.001 \mu \mathrm{M}$; p: 0.014 for $0.01 \mu \mathrm{M}$; p: 0.000 for $0.1 \mu \mathrm{M}$; p: 0.000 for $1 \mu \mathrm{M}$ ) (Figure 1).

\subsection{PKC was involved in the irisin-induced relaxation of} rat thoracic aortas

Incubation of tissues with PKC inhibitor BIM I (150 nM) for 20 min caused a significant change in the relaxation responses to irisin. BIM I incubation resulted in a statistically significant decrease in the relaxation responses of irisin applied at cumulative concentrations of $0.01,0.1$, and $1 \mu \mathrm{M}$ ( $\mathrm{p}$ : 0.039 for $0.01 \mu \mathrm{M}$; $\mathrm{p}: 0.000$ for $0.1 \mu \mathrm{M}$; $\mathrm{p}$ : 0.000 for $1 \mu \mathrm{M})$. BIM I almost completely inhibited relaxation responses to irisin (Figure 2).

3.3. DMSO did not modulate the irisin-induced vasorelaxation effects

Incubation of tissues with DMSO $(50 \mu \mathrm{L})$ for $10 \mathrm{~min}$ did not cause a significant change in the relaxation responses to irisin. DMSO incubation at solvent concentration did not cause a remarkable change on any of the relaxation responses of irisin applied at cumulative concentrations of $0.01,0.1$, and $1 \mu \mathrm{M}$ (p: 1.000 for $0.01 \mu \mathrm{M}$; p: 1.000 for 0.1 $\mu \mathrm{M}$; p: 1.000 for $1 \mu \mathrm{M}$ ). DMSO did not significantly affect the relaxation responses to irisin (Figure 3) (Table).

\section{Discussion}

The aim of the present study was to investigate the functional relaxant effects of irisin on rat thoracic aorta and its underlying mechanisms. We have shown that irisin can significantly induce concentration-dependent relaxations in endothelium-intact rat thoracic aorta and the vasorelaxant effects of irisin were associated with PKC. The results suggest that irisin-induced relaxation responses are regulated probably via activating signaling pathways implicating PKC.

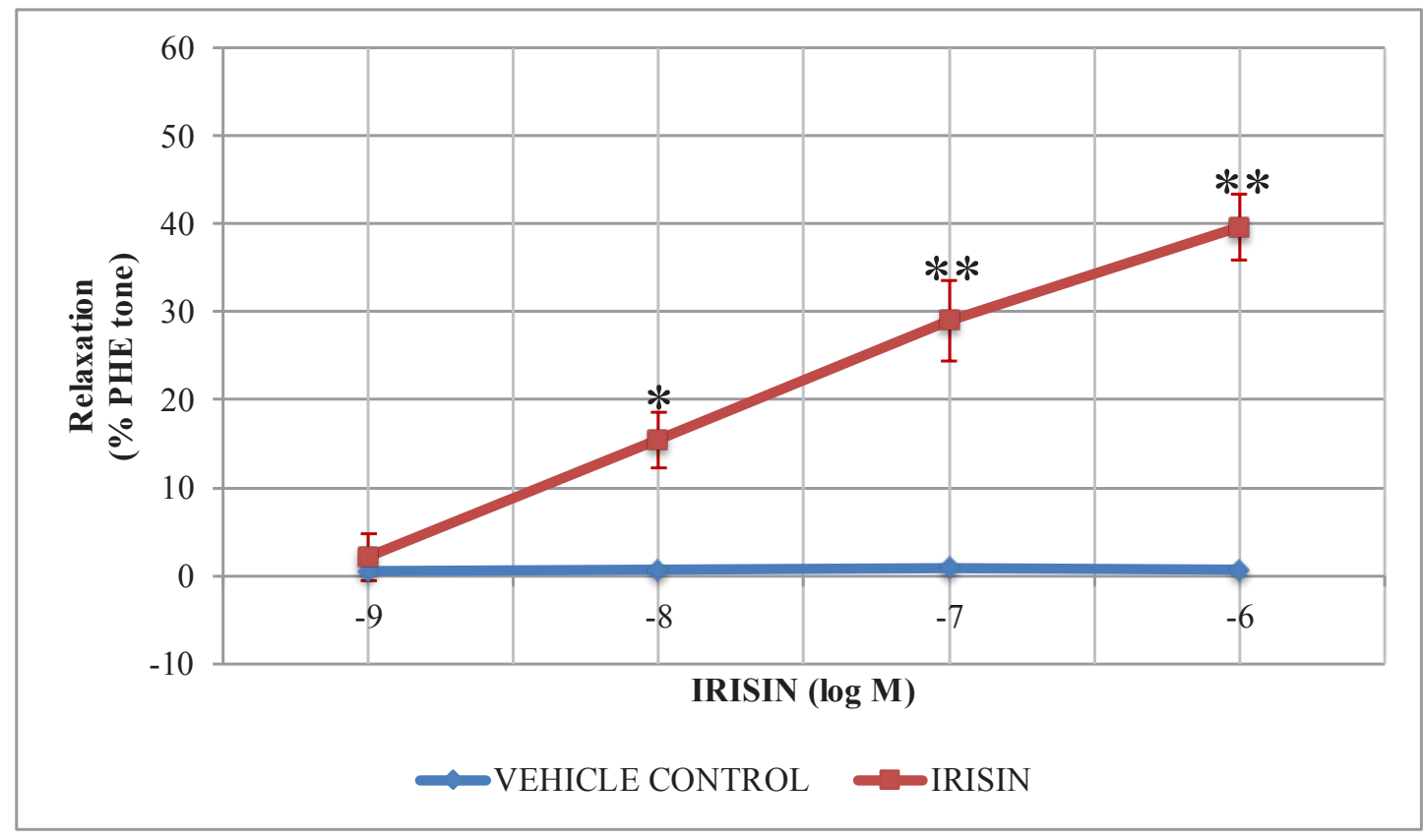

Figure 1. Concentration-dependent relaxant effects of irisin on PHE precontracted endothelium-intact rat thoracic aorta rings. The vehicle control group was treated with distilled water. The relaxant effects of irisin on thoracic aorta rings were calculated as a percentage of the contraction in response to $10 \mu \mathrm{M}$ PHE. The data were expressed as the mean \pm SD. $(n=8){ }^{*}: p<0.05$ vs. control. ${ }^{*}: p<0.001$ vs. control. 


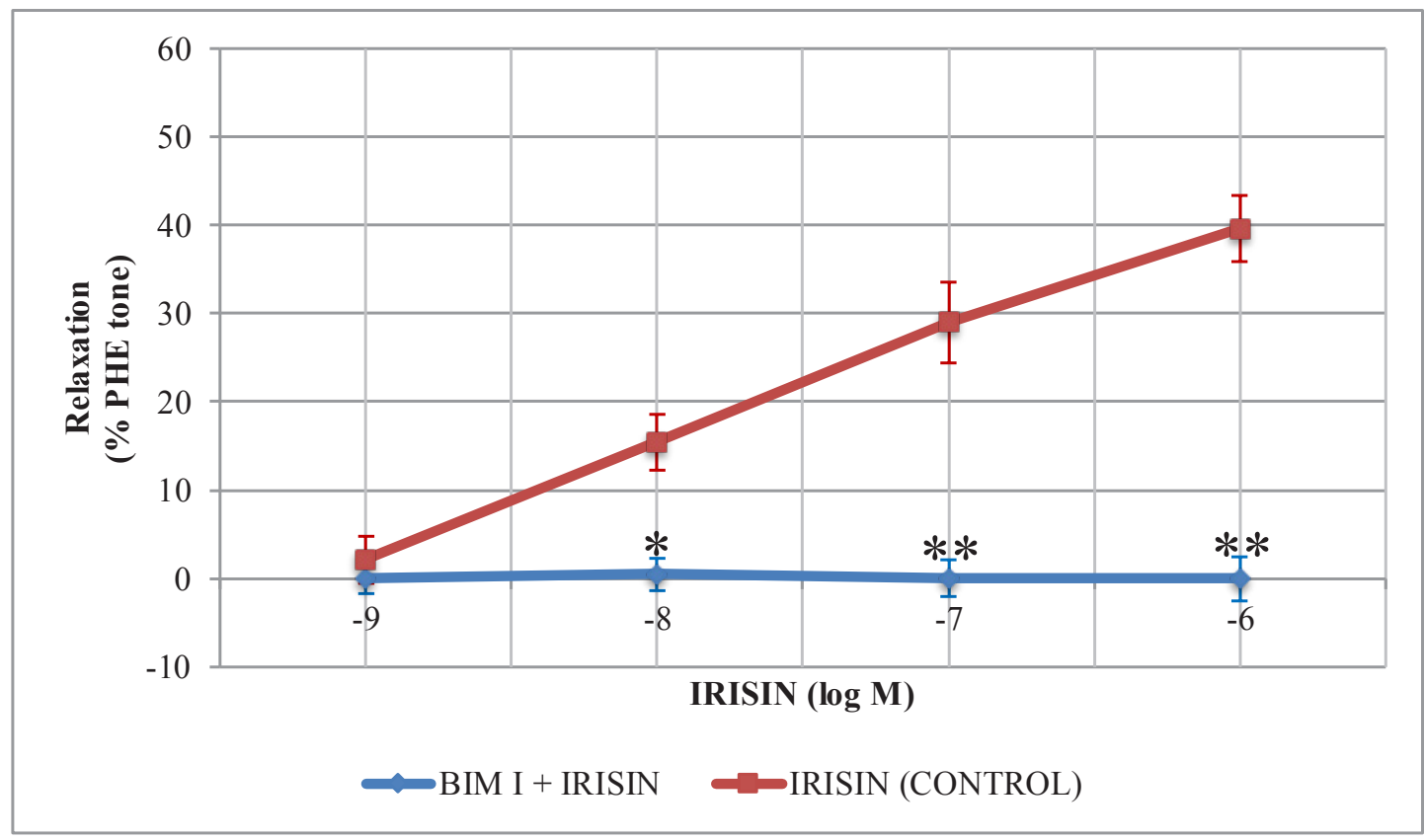

Figure 2. Effects of PKC inhibitor BIM I on irisin-induced relaxation in rat thoracic aorta. Relaxant effects of irisin on endothelium-intact thoracic aorta rings were measured in the absence (control) or presence of BIM I. The relaxant effects were calculated as a percentage of the contraction in response to $10 \mu \mathrm{M}$ PHE. The data were expressed as the mean \pm SD. $(n=8) .^{*}: p<0.05$ vs. control. ${ }^{*}: p<0.001$ vs. control.

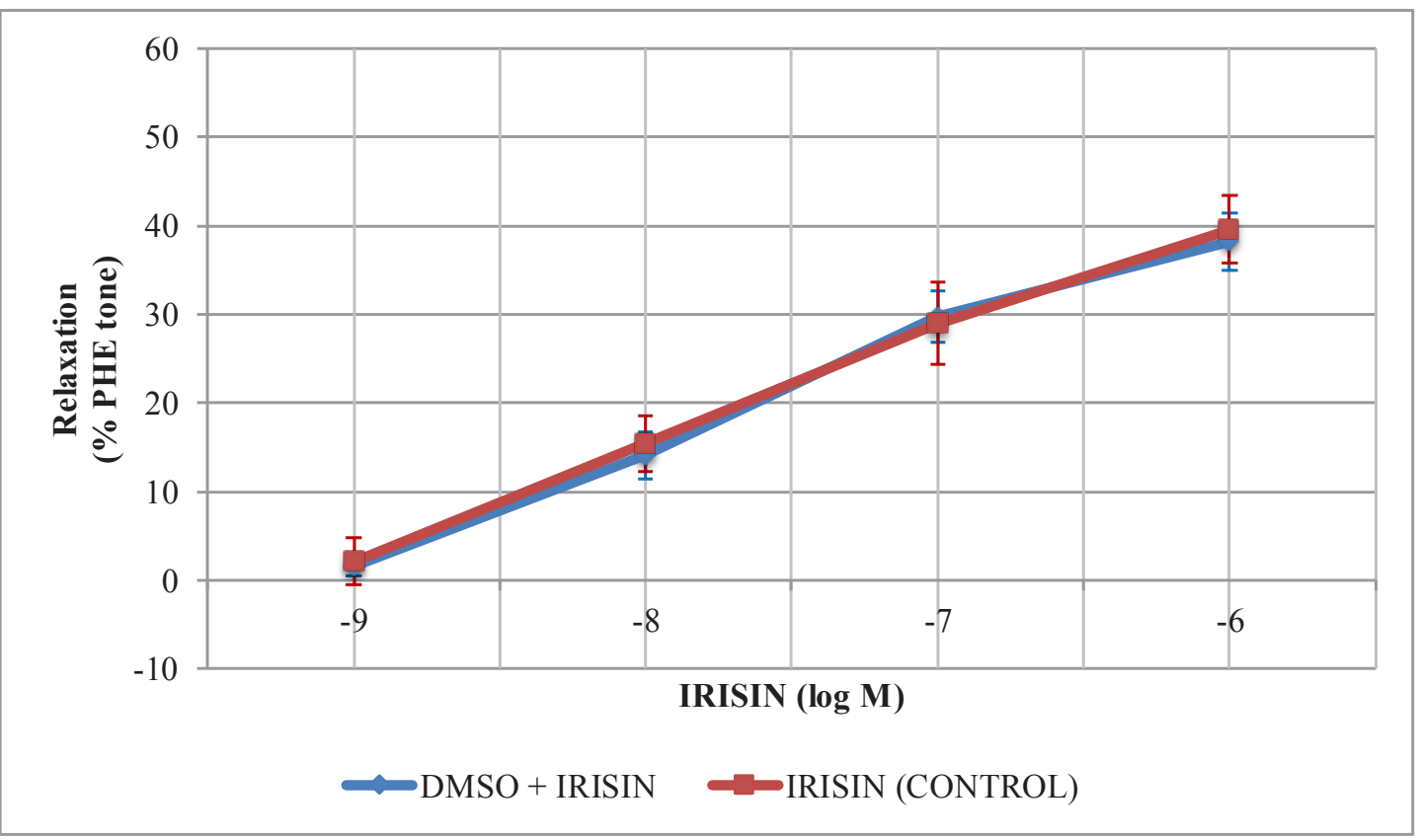

Figure 3. Effects of DMSO (at the solvent concentration of $50 \mu \mathrm{L}$ ) on irisin-induced relaxation in rat thoracic aorta. Relaxant effects of irisin on endothelium-intact thoracic aorta rings were measured in the absence (control) or presence of DMSO. The relaxant effects were calculated as a percentage of the contraction in response to $10 \mu \mathrm{M}$ PHE. The data were expressed as the mean \pm SD $(n=8)$. 
Table. Descriptive statistics for each group. Outliers have been corrected.

\begin{tabular}{|c|c|c|c|c|c|c|}
\hline & \multirow{3}{*}{$\begin{array}{l}\text { Concentration } \\
(\log M)\end{array}$} & \multirow{3}{*}{ Mean } & \multirow{3}{*}{ Median } & \multirow{3}{*}{$\begin{array}{l}\text { Std. } \\
\text { Deviation }\end{array}$} & \multicolumn{2}{|c|}{ Test of Normality } \\
\hline & & & & & \multicolumn{2}{|c|}{ Shapiro-Wilk } \\
\hline & & & & & Statistic & $\mathbf{p}$ \\
\hline \multirow{4}{*}{$\begin{array}{l}\text { VEHICLE } \\
\text { CONTROL } \\
(\mathbf{n}=\mathbf{8})\end{array}$} & -9 & 0.50 & 0.00 & 0.00 & 0.724 & 0.004 \\
\hline & -8 & 0.62 & 0.00 & 0.00 & 0.693 & 0.002 \\
\hline & -7 & 0.87 & 0.50 & 0.01 & 0.809 & 0.036 \\
\hline & -6 & 0.62 & 0.00 & 0.00 & 0.693 & 0.002 \\
\hline \multirow{4}{*}{$\begin{array}{l}\text { IRISIN } \\
(n=8)\end{array}$} & -9 & 2.12 & 1.50 & 2.66 & 0.945 & 0.662 \\
\hline & -8 & 15.50 & 15.00 & 3.16 & 0.957 & 0.785 \\
\hline & -7 & 29.00 & 29.50 & 4.62 & 0.943 & 0.644 \\
\hline & -6 & 39.62 & 39.50 & 3.77 & 0.954 & 0.747 \\
\hline \multirow{4}{*}{$\begin{array}{l}\text { BIM-I } \\
(\mathrm{n}=8)\end{array}$} & -9 & 0.00 & 0.50 & 1.71 & 0.929 & 0.503 \\
\hline & -8 & 0.50 & 2.50 & 1.80 & 0.786 & 0.020 \\
\hline & -7 & 0.00 & 0.50 & 2.06 & 0.911 & 0.361 \\
\hline & -6 & 0.00 & 0.50 & 2.47 & 0.894 & 0.255 \\
\hline \multirow{4}{*}{$\begin{array}{l}\text { DMSO } \\
(\mathrm{n}=\mathbf{8})\end{array}$} & -9 & 1.62 & 2.00 & 1.08 & 0.875 & 0.168 \\
\hline & -8 & 14.12 & 13.50 & 2.70 & 0.913 & 0.378 \\
\hline & -7 & 29.75 & 30.00 & 2.85 & 0.952 & 0.727 \\
\hline & -6 & 38.25 & 39.50 & 3.26 & 0.913 & 0.377 \\
\hline
\end{tabular}

Despite great advances in medicine, proper treatment of arterial hypertension, diabetes mellitus, and chronic kidney disease remains a major challenge. Undiagnosed and untreated arterial hypertension or diabetes mellitus may lead to the development of chronic kidney disease and, consequently, to cardiovascular disorders [8].

The frequency of insulin resistance, obesity, and type II diabetes mellitus, and cardiovascular diseases such as hypertension and atherosclerosis due to these diseases has increased. Atherosclerosis, the main cause of coronary artery disease and stroke, remains the primary cause of global mortality $[9,10]$.

Skeletal muscles have been defined as the endocrine organ in recent years [11]. Cytokines known as myokines, released from skeletal muscles during and/or immediately after exercise, mediate the beneficial effects of exercise on metabolism and the cardiovascular system $[12,13]$.

Irisin, a newly described myokine, is increased with exercise and has the potential to play a preventive and/or therapeutic role in the management of the aforementioned chronic diseases [1,14,15-20]. In this context, it is considered that irisin may be an important therapeutic target in the prevention of the development of vascular pathologies and the treatment of atherosclerotic cardiovascular diseases. The precise functions of irisin related to the vascular tissue have not yet been clarified.
However, few current studies have focused on the effects of irisin on vascular activity and the mechanisms involved.

Zhang et al. [15] have found that central administration of irisin increases blood pressure and cardiac contractility. In contrast, peripheral administration of irisin reduces blood pressure in both control and spontaneously hypertensive rats. Irisin dilates mesenteric artery rings through ATP-sensitive potassium channels. Han et al. [16] have provided the first evidence that irisin improves endothelial function in aortas of high-fat-dietinduced obese mice. The mechanism for this protective effect is related to the activation of the 5 adenosine monophosphate-activated protein kinase (AMPK)endothelial nitric oxide synthase (eNOS) signaling pathway. Jiang et al. [17] have investigated the effects of irisin on vascular activity and the mechanisms involved in mouse mesenteric arteries. They reported that irisin induced both endothelium-dependent and -independent relaxation in a dose-dependent manner. Endotheliumdependent relaxation is mediated by the nitric oxide (NO)cyclic guanosine monophosphate (cGMP) dependent pathway, while endothelium-independent relaxation may be influenced by reducing $\mathrm{Ca}^{2+}$ influx through inhibiting voltage-dependent $\mathrm{Ca}^{2+}$ channels and intracellular $\mathrm{Ca}^{2+}$ release through both inositol-trisphosphate receptor $\left(\mathrm{IP}_{3} \mathrm{R}\right)$ and ryanodine receptor $(\mathrm{RyR})$ channels. Fu et al. 
[18] have concluded that acute administration of irisin increases endothelium-dependent vasodilation and lowers the blood pressure of spontaneously hypertensive rats by amelioration of endothelial dysfunction of the mesenteric artery through the AMPK-protein kinase B (Akt)-eNOS-NO signaling pathway. Hou et al. [19] have shown that irisin improves the anticontractile properties of perivascular adipose tissue (PVAT) from the thoracic aorta in diet-induced obese mice. The mechanism for protective effects of irisin appears to be related to upregulation of the heme oxygenase-1/adiponectin axis in PVAT and browning of PVAT. Ye et al. [20] have stated that irisin-induced endothelium-dependent vasodilation is related to the stimulation of extracellular $\mathrm{Ca}^{2+}$ influx via transient receptor potential vanilloid subtype 4 (TRPV4) channels in rat mesenteric arteries.

PKC is a key regulatory enzyme involved in the transduction of signals in several cellular functions in vascular smooth muscle cells, including vascular smooth muscle contractility [21]. PKC regulates the contractility of vascular smooth muscle cells in various pathways, including changes in myofilaments $\mathrm{Ca}^{2+}$-sensitivity and regulation of intracellular free calcium concentration $\left(\left[\mathrm{Ca}^{2+}\right]_{i}\right.$, as well as by reducing endothelium-mediated vasodilation [22-24]. This enzyme is also involved in the regulation of the plasmalemma ion permeability in vascular smooth muscle cells. For instance, PKC has been shown to regulate the activity of $\mathrm{K}^{+}$channels. PKC can influence vascular activity by physiological changes both in $\mathrm{Ca}^{2+}$ concentrations and membrane potential. The increased reactivity of the vascular wall is also associated with blunted endothelium-dependent responses due to reduced sensitivity to NO. Briefly, increased vascular smooth muscle cells myofilaments $\mathrm{Ca}^{2+}$ sensitivity and decreased sensitivity to $\mathrm{NO}$ can both be the result of a change in PKC activity [25]. The demonstration of a recent study that irisin suppresses oxidative/nitrative stresses by inhibiting PKC- $\beta$-related pathways in human umbilical vein endothelial cells cultured in high-glucose/high-fat [26] suggests that the vasodilator effect of irisin may also be due to the inhibition of signaling pathways implicating PKC. Although it is commonly accepted that PKC signaling inhibits eNOS activity, the distinct role of different PKC isoforms has not been studied so far. The PKC family comprises roughly 12 different isozymes that activate distinct downstream pathways. In this regard, activation of PKCa leads to phosphorylation of calponin, an actinrelated regulatory protein in smooth muscle cells, resulting in vascular smooth muscle contraction; on the other hand, it can cause positive modulation of blood flow through the activation of eNOS in the endothelium $[27,28]$.

In the present study, we investigated the role of PKC on the concentration-dependent relaxation responses of irisin. The vasorelaxant effects of irisin were significantly attenuated by pretreatment with PKC inhibitor BIM I, suggesting that signaling pathways implicating PKC may be associated with irisin-induced vasodilation. Thus, our results demonstrate for the first time that PKC stimulates irisin-induced vasodilation in rat thoracic aorta. However, PKC isoforms may have opposite effects on irisin-induced vasodilation. Therefore, further study is necessary to examine the hypothesis that irisin induces relaxation by enhancing the PKC-mediated nitric oxide synthesis in endothelium and/or inhibiting the PKC-regulated Ca sensitivity in vascular smooth muscle. Besides, further research using specific activators and inhibitors is required to clarify this effect.

Differences could be observed in the vasodilator activity of irisin on different vascular preparations obtained from various species. It is thought that this may be due to methodological differences such as the species from which the tissues were removed, the vascular tissues in which the experiments were performed (locations and diameters of vascular tissues), and/or the agents used to induce precontraction. Vasoreactivities of the arteries may be different from each other. It is known that the density and types of ion transport mechanisms and/or physiological/ pharmacological receptors, as well as differences in substances released from the endothelium, could have an important role in changes in the responses of vascular smooth muscles to various agents $[29,30]$. For instance, the effects of hyperpolarizing factors increase inversely with vessel diameter [31]. Therefore, in the present study, the relaxing effects of irisin were investigated in thoracic aorta preparations isolated from Wistar Albino rats. Furthermore, the possible role of PKC regarding action mechanisms of irisin assessed in this study has not been performed on any species and/or vascular tissues before.

In this context, our results showed that $0.01,0.1$, and 1 $\mu \mathrm{M}$ concentrations of irisin have a vasodilatative effect on rat thoracic aorta, a conducting artery. Thus, we reported that irisin has relaxing effects on vascular smooth muscle contractility both at mean plasma concentration (0.1 $\mu \mathrm{M})$ [18] and a wide range of concentrations, including the upper and lower levels of mean concentration (0.01$1 \mu \mathrm{M})$. Pretreatment with DMSO, a solvent of BIM I, did not modulate irisin-induced relaxation. It was further demonstrated that the irisin-induced vasorelaxation effects on endothelium-intact rat thoracic aorta were significantly abolished by pretreatment with PKC inhibitor BIM I. Thus, this study provides the first evidence that irisin-induced vasodilation is regulated probably via activating signaling pathways implicating PKC.

In conclusion, this study demonstrated that irisin induces concentration-dependent relaxation responses in endothelium-intact rat thoracic aorta rings. Furthermore, 
the present study is the first to report that the mechanism for this vasorelaxant effect is associated with the activity of PKC. These results suggest that irisin might be a useful agent in the treatment of abnormal vasoconstrictionrelated diseases such as hypertension and atherosclerosis, in addition to its key role in regulating energy metabolism, resulting in thermogenesis and weight loss, by increasing uncoupling protein-1 (UCP-1) expression in adipose tissue. This study further supports the beneficial effect of exercise on vascular function, depending on the release of irisin, an exercise hormone.

However, the findings presented in our study are preliminary. The precise roles of signaling pathways implicating PKC in irisin-mediated vasodilation need to be further elucidated in clinically relevant animal models.

\section{References}

1. Boström P, Wu J, Jedrychowski MP, Korde A, Ye L et al. A PGC1a-dependent myokine that drives brown-fat-like development of white fat and thermogenesis. Nature 2012; 481: 463-468. doi: 10.1038/nature10777

2. Sanchis-Gomar F, Lippi G, Mayero S, Perez-Quilis C, GarcíaGiménez JL. Irisin: a new potential hormonal target for the treatment of obesity and type 2 diabetes. Journal of Diabetes, 2012; 4: 196. doi: 10.1111/j.1753-0407.2012.00194.x

3. Polyzos SA, Anastasilakis AD, Efstathiadou ZA, Makras P, Perakakis N et al. Irisin in metabolic diseases. Endocrine 2018; 59: 260-274. doi: 10.1007/s12020-017-1476-1

4. Korta P, Pocheć E, Mazur-Biały A. Irisin as a multifunctional protein: implications for health and certain diseases. Medicina (Kaunas) 2019; 55: 485. doi: 10.3390/medicina55080485

5. Ma C, Ding H, Deng Y, Liu H, Xiong X et al. Irisin: a new code uncover the relationship of skeletal muscle and cardiovascular health during exercise. Frontiers in Physiology 2021; 12: 620608. doi: 10.3389/fphys.2021.620608

6. Brunner H, Cockcroft JR, Deanfield J, Donald A, Ferrannini E et al. Endothelial function and dysfunction. Part II: association with cardiovascular risk factors and diseases. A statement by the Working Group on Endothelins and Endothelial Factors of the European Society of Hypertension. Journal of Hypertension 2005; 23: 233-246. doi: 10.1097/00004872-200502000-00001

7. Jespersen B, Tykocki NR, Watts SW, Cobbett PJ. Measurement of smooth muscle function in the isolated tissue bath-applications to pharmacology research. Journal of Visualized Experiments 2015; 19: 52324. doi: 10.3791/52324

8. Maciorkowska M, Musiałowska D, Małyszko J. Adropin and irisin in arterial hypertension, diabetes mellitus and chronic kidney disease. Advances in Clinical and Experimental Medicine 2019; 28: 1571-1575. doi: 10.17219/acem/104551

9. Byun $\mathrm{K}$, Lee $\mathrm{S}$. The potential role of irisin in vascular function and atherosclerosis: a review. International Journal of Molecular Sciences 2020; 21: 7184. doi: 10.3390/ijms21197184
Besides, more detailed mechanism studies and in vivo studies are necessary to establish the efficacy of irisin in the management and treatment of cardiovascular diseases.

\section{Acknowledgment/Disclaimers/Conflict of interest}

This work was supported by the Scientific and Technological Research Council of Turkey [project no. 219S306].

All authors declare that there is no conflict of interest that may have influenced either the conduct or the presentation of the research.

\section{Informed consent}

This study was approved by the decision of Bursa Uludağ University Animal Experiments Local Ethics Committee dated 03/04/2019 and numbered 2019-04/10.

10. Voutyritsa E, Kyriakos G, Patsouras A, Damaskos C, Garmpi A et al. Experimental agents for the treatment of atherosclerosis: new directions. Journal of Experimental Pharmacology 2021; 13: 161-179. doi: 10.2147/JEP.S265642

11. Pedersen BK, Akerström TC, Nielsen AR, Fischer CP. Role of myokines in exercise and metabolism. Journal of Applied Physiology 2007; 103: 1093-1098. doi: 10.1152/ japplphysiol.00080.2007

12. Pedersen BK, Febbraio MA. Muscles, exercise and obesity: skeletal muscle as a secretory organ. Nature Reviews Endocrinology 2012; 8: 457-465. doi: 10.1038/nrendo.2012.49

13. Strasser B. Physical activity in obesity and metabolic syndrome. Annals of the New York Academy of Sciences 2013; 1281: 141159. doi: 10.1111/j.1749-6632.2012.06785.x

14. Demirel S, Şahintürk S, Özyener F. Irisin and its effects on vascular contractility. Medical Journal of Suleyman Demirel University 2021; 28: 163-170. doi: 10.17343/sdutfd.718412

15. Zhang W, Chang L, Zhang C, Zhang R, Li Z et al. Central and peripheral irisin differentially regulate blood pressure. Cardiovascular Drugs and Therapy 2015; 29: 121-127. doi: 10.1007/s10557-015-6580-y

16. Han F, Zhang S, Hou N, Wang D, Sun X. Irisin improves endothelial function in obese mice through the AMPK-eNOS pathway. American Journal of Physiology-Heart and Circulatory 2015; 309: H1501-H1508. doi: 10.1152/ajpheart.00443.2015

17. Jiang M, Wan F, Wang F, Wu Q. Irisin relaxes mouse mesenteric arteries through endothelium-dependent and endotheliumindependent mechanisms. Biochemical and Biophysical Research Communications 2015; 468: 832-836. doi: 10.1016/j. bbrc.2015.11.040

18. Fu J, Han Y, Wang J, Liu Y, Zheng S et al. Irisin lowers blood pressure by improvement of endothelial dysfunction via AMPK-Akt-eNOS-NO pathway in the spontaneously hypertensive rat. Journal of the American Heart Association 2016; 5: e003433. doi: 10.1161/JAHA.116.003433 
19. Hou N, Liu Y, Han F, Wang D, Hou X et al. Irisin improves perivascular adipose tissue dysfunction via regulation of the heme oxygenase-1/adiponectin axis in diet-induced obese mice. Journal of Molecular and Cellular Cardiology 2016; 99: 188-196. doi: 10.1016/j.yjmcc.2016.09.005

20. Ye L, Xu M, Hu M, Zhang H, Tan X et al. TRPV4 is involved in irisin-induced endothelium-dependent vasodilation. Biochemical and Biophysical Research Communications 2017; 495: 41-45. doi: 10.1016/j.bbrc.2017.10.160

21. Cosentino-Gomes D, Rocco-Machado N, Meyer-Fernandes JR. Cell signaling through protein kinase $\mathrm{C}$ oxidation and activation. International Journal of Molecular Sciences 2012; 13: 10697-10721. doi: 10.3390/ijms130910697

22. Somlyo AP, Somlyo AV. Ca2+ sensitivity of smooth muscle and nonmuscle myosin II: modulated by $\mathrm{G}$ proteins, kinases, and myosin phosphatase. Physiological Reviews 2003; 83: 13251258. doi: $10.1152 /$ physrev.00023.2003

23. Massett MP, Ungvari Z, Csiszar A, Kaley G, Koller A. Different roles of PKC and MAP kinases in arteriolar constrictions to pressure and agonists. American Journal of Physiology-Heart and Circulatory 2002; 283: H2282-H2287. doi: 10.1152/ ajpheart.00544.2002

24. Korzick DH, Laughlin MH, Bowles DK. Alterations in PKC signalling underlie enhanced myogenic tone in exercisetrained porcine coronary resistance arteries. Journal of Applied Physiology 2004; 96: 1425-1432. doi: 10.1152/ japplphysiol.01077.2003

25. Soloviev AI, Kizub IV. Mechanisms of vascular dysfunction evoked by ionizing radiation and possible targets for its pharmacological correction. Biochemical Pharmacology 2018; 159: 121-139. doi: 10.1016/j.bcp.2018.11.019
26. Zhu D, Wang H, Zhang J, Zhang X, Xin C et al. Irisin improves endothelial function in type 2 diabetes through reducing oxidative/nitrative stresses. Journal of Molecular and Cellular Cardiology 2015; 87: 138-147. doi: 10.1016/j.yjmcc.2015.07.015

27. Partovian C, Zhuang Z, Moodie K, Lin M, Ouchi N et al. PKCalpha activates eNOS and increases arterial blood flow in vivo. Circulation Research 2005; 97: 482-487. doi: 10.1161/01. RES.0000179775.04114.45

28. Wang Y, Zhou H, Wu B, Zhou Q, Cui D et al. Protein kinase C isoforms distinctly regulate propofol-induced endotheliumdependent and endothelium-independent vasodilation. Journal of Cardiovascular Pharmacology 2015; 66: 276-284. doi: 10.1097/FJC.0000000000000275

29. Clark SG, Fuchs LC. Role of nitric oxide and $\mathrm{Ca}^{++}$-dependent $\mathrm{K}^{+}$channels in mediating heterogeneous microvascular responses to acetylcholine in different vascular beds. Journal of Pharmacology and Experimental Therapeutics 1997; 282: 1473-1479. PMID: 9316861

30. Mulvany MJ, Aalkjaer C. Structure and function of small arteries. Physiological Reviews 1990; 70: 921-961. doi: 10.1152/ physrev.1990.70.4.921

31. de Wit C, Wölfle SE. EDHF and gap junctions: important regulators of vascular tone within the microcirculation. Current Pharmaceutical Biotechnology 2007; 8: 11-25. doi: $10.2174 / 138920107779941462$ 